\title{
A model for reactive porous transport during re-wetting of hardened concrete
}

\author{
Michael Chapwanya ${ }^{\dagger} \quad$ John M. Stockie ${ }^{\dagger} \S \quad$ Wentao Liu ${ }^{\ddagger}$
}

June 7, 2021

\begin{abstract}
A mathematical model is developed that captures the transport of liquid water in hardened concrete, as well as the chemical reactions that occur between the imbibed water and the residual calcium silicate compounds residing in the porous concrete matrix. The main hypothesis in this model is that the reaction product - calcium silicate hydrate gel - clogs the pores within the concrete thereby hindering water transport. Numerical simulations are employed to determine the sensitivity of the model solution to changes in various physical parameters, and compare to experimental results available in the literature.
\end{abstract}

\section{Introduction}

Concrete is a ubiquitous construction material that derives its utility from a combination of strength, versatility and relatively low cost. In fact, concrete is the second most consumed material on the planet next to water [47. The primary ingredients that go into the making of concrete are water, Portland cement (a fine powder consisting primarily of calcium silicate compounds), and solid aggregates such as sand and gravel. When mixed together, these ingredients undergo a complex physico-chemical transformation which can be divided into a number of discrete steps: an initial hydration stage that occurs over a period of hours or days; a drying/curing period that can require months or even years to complete; and additional reactions arising from carbonation and various degradation processes that typically also occur over very long time periods.

Mathematical modelling of transport and reaction in concrete has been the subject of a large number of papers in the scientific and engineering literature. The earliest study that we are aware of which treats water transport in concrete as a nonlinear diffusion process is that of Bažant and Najjar [6]. Later work considered the additional effect of transport and reaction of chemical species in

\footnotetext{
${ }^{\dagger}$ Department of Mathematics, Simon Fraser University, Burnaby, BC, Canada.

$\ddagger$ Department of Applied Mathematics, University of Waterloo, Waterloo, ON, Canada.

$\S$ Corresponding author (stockie@math.sfu.ca).
} 
the context of initial cement hydration [13, 41, [5] or concrete carbonation [38, 37, 19. We remark that most of these models assume a constant porosity even though experimental evidence overwhelmingly suggests that the pore structure varies significantly over time owing to reactant consumption, crystallization, and swelling of products throughout the various stages of concrete hydration [38, 23, 43. In fact, the only models we are aware of that allow for a variable porosity are in the context of carbonation, where Meier et al. [37. specify the porosity as a given decaying exponential function of time, while Bary and Sellier [5] allow the porosity to depend on the solution via changes in the pore volume from solidified reaction products.

We focus here on a later stage in the life of concrete, namely the process of re-wetting or "secondary hydration" in which hardened and cured concrete experiences imbibition of water, due to periodic rainfall for example. The proportion of reactive silicates in the cement that are consumed during the initial hydration reactions (called the degree of hydration) is typically on the order of $50 \%$ [9; consequently, there are significant levels of residual reactants remaining in hardened concrete and so the effect of secondary reactions occurring during re-wetting cannot be ignored. This study is motivated by the experimental work of Barrita et al. [4, 3] and Taylor et al. [43] who placed dry concrete samples in a liquid bath and carefully observed the progress of the subsequent wetting front. They found that when a non-reactive liquid such as isopropanol was used, the front speed was proportional to the square root of time as predicted by nonlinear diffusion analysis. When water was used instead, the wetting front moved more slowly than the theory predicted and in some cases stalled completely - an effect that is usually referred to as anomalous diffusion. Hall [24] has suggested that this effect is due to physico-chemical interactions between the wetting fluid and the porous solid. It is natural to hypothesize therefore that the reduction in wetting front speed arises from residual calcium silicates in the porous matrix reacting with water to form calcium silicate hydrate or C-S-H, which precipitates in the form of a gel that clogs the pores in the concrete; this hypothesis is supported by the results in 23 .

Observations of anomalous diffusion have been reported in 32 where the authors proposed instead that deviations in wetting front speed can be modeled using a modified (non-Darcian) porous transport equation. This approach provides a reasonable match with experiments and gives rise to a new and potentially interesting class of nonlinear diffusion equations and scaling laws; however, there is no direct support for this model in terms of a physical mechanism for concrete hydration. In a related study [35, another model is proposed that includes an explicit time-dependence in the water diffusion coefficient. They showed that by assuming the cumulative deposition follows a power law in time, they could reproduce similar clogging results; unfortunately, it is not at all clear how one would obtain the power law coefficients in a given wetting scenario.

Some authors have addressed clogging phenomena in the context of concrete carbonation, such as Saetta et al. [42] who incorporated a functional dependence on the carbonate concentration into the transport coefficients of their model. Meier et al. 37] also employed an empirical approach, but instead they 
assumed the porosity decays as a given exponential function of time, which has the disadvantage that there is no direct coupling between water transport and the precipitated reaction products that are causing the actual clogging. Related work on self-desiccation (or internal drying) during initial hydration and its connection with autogenous shrinkage have been studied using pore-level microstructure simulations [10].

In this paper, we develop a model that aims to test the hypothesis that incorporating the chemistry of residual cement constituents and the effect of the resulting C-S-H gel formation on pore structure can explain the apparent clogging effects observed in concrete re-wetting experiments. We begin in Section 2 with a brief overview of cement chemistry and the physico-chemical changes that occur in cement during hydration. We develop the mathematical model in Section 3 using a macroscopic approach that is motivated by the clogging models developed in the bioremediation literature (see for example [16]) wherein the accumulation of biomass - analogous to cement hydration products - is responsible for the reduction in porosity. Numerical simulations of the resulting system of nonlinear partial differential equations are performed in Section 4 and the results are compared with experiments. We show that our model captures observed clogging behaviour both qualitatively and quantitatively with a minimum of parameter fitting, and we explain in Section 5 how these results might be generalized in future to handle a range of other related phenomena in concrete transport.

\section{Overview of cement chemistry}

While this paper is not concerned directly with the primary hydration of cement, the same hydration reactions occur during the re-wetting phase when residual unhydrated silicates remaining in the hardened concrete matrix are exposed to water. We will therefore begin by presenting some background information on the process of cement hydration that is drawn largely from [33, 11]. Portland cement is the key binding agent in concrete and has as its major constituents tricalcium silicate $\left(3 \mathrm{CaO} \cdot \mathrm{SiO}_{2}\right.$, commonly referred to as alite) and dicalcium silicate $\left(2 \mathrm{CaO} \cdot \mathrm{SiO}_{2}\right.$, known as belite) which make up approximately $50 \%$ and $25 \%$ respectively of dry cement by mass. The remaining $25 \%$ consists primarily of tricalcium aluminate, tetracalcium aluminoferrite, and gypsum, with smaller amounts of certain other admixtures whose purpose is to influence such properties as strength, flexibility, setting time, etc. In this paper we will concentrate solely on the two primary constituents, alite and belite.

The cement powder is mixed with water and aggregates (sand, gravel and crushed stone) to make a workable paste that can then be easily poured or molded and left to harden. During the initial hydration stage, the silicates dissolve in and react with the water to form calcium hydroxide or $\mathrm{Ca}(\mathrm{OH})_{2}$, and calcium silicate hydrate or C-S-H; the latter notation does not denote a specific chemical compound but rather represents a whole family of hydrates having $\mathrm{Ca} / \mathrm{Si}$ ratios that range between 0.6 and 2.0. A significant amount of heat is 
released during the conversion of alite and belite into C-S-H since the hydration reactions are exothermic. Calcium hydroxide and C-S-H precipitate out of solution in crystalline form, and these solid precipitates then act as nucleation sites that further enhance formation of C-S-H. It is the crystalline or gel form of C-S-H that is ultimately responsible for the strength of concrete.

The hydration process can proceed via several possible reactions, but we restrict ourselves to a particular reaction sequence that is employed in both [41] and [46. The mechanism for alite hydration begins with a dissolution phase

$$
3 \mathrm{CaO} \cdot \mathrm{SiO}_{2}+3 \mathrm{H}_{2} \mathrm{O} \stackrel{r_{1}}{\longrightarrow} 3 \mathrm{Ca}^{2+}+4 \mathrm{OH}^{-}+\mathrm{H}_{2} \mathrm{SiO}_{4}^{2-},
$$

followed by a reaction in solution to form aqueous C-S-H

$$
\mathrm{H}_{2} \mathrm{SiO}_{4}^{2-}+\frac{3}{2} \mathrm{Ca}^{2+}+\mathrm{OH}^{-} \stackrel{r_{2}}{\longrightarrow} \text { C-S-H }(a q),
$$

and precipitation of calcium hydroxide according to

$$
\mathrm{Ca}^{2+}+2 \mathrm{OH}^{-} \stackrel{r_{3}}{\longrightarrow} \mathrm{Ca}(\mathrm{OH})_{2}
$$

In each chemical formula we have indicated the rate of the reaction by $r_{i}\left[d a y^{-1}\right]$ for $i=1,2,3$.

For the remainder of this paper, we adopt the cement chemistry convention in which the following abbreviations are used: $\mathrm{C}=\mathrm{CaO}, \mathrm{S}=\mathrm{SiO}_{2}$ and $\mathrm{H}=\mathrm{H}_{2} \mathrm{O}$. Then the overall reaction, leaving out intermediate ionic species, can be written in terms of the single formula

$$
2 \mathrm{C}_{3} \mathrm{~S}+6 \mathrm{H} \stackrel{r_{\alpha}}{\longrightarrow} \mathrm{C}-\mathrm{S}-\mathrm{H}(a q)+3 \mathrm{CH},
$$

where $r_{\alpha}$ represents an overall rate constant for alite. Motivated by the models developed in [38, 37, 11, we consider only the simplified kinetics represented by (11). We also take the chemical form of C-S-H to be that of $\mathrm{C}_{3} \mathrm{~S}_{2} \mathrm{H}_{3}$, that because of the amorphous nature of C-S-H can only be true in some averaged sense. A similar formula holds for belite

$$
2 \mathrm{C}_{2} \mathrm{~S}+4 \mathrm{H} \stackrel{r_{\beta}}{\longrightarrow} \mathrm{C}-\mathrm{S}-\mathrm{H}(a q)+\mathrm{CH},
$$

where we note that $r_{\alpha} \gg r_{\beta}$ [13, 38, 9]. Alite is also mainly responsible for the early stage strength of the concrete (through approximately the first seven days) while belite contributes to the later strength.

Following [46], we include a precipitation (or deposition) step in which the aqueous C-S-H product precipitates out of solution to bind with the porous matrix:

$$
\text { C-S-H }(a q) \underset{k_{\text {diss }}}{\stackrel{k_{\text {prec }}}{\rightleftharpoons}} \text { C-S-H }(g e l) \text {, }
$$

where the rate of precipitation is denoted by $k_{\text {prec }}\left[\right.$ day $\left.^{-1}\right]$. We allow for a dissolution process with rate constant $k_{\text {diss }}$, although in most of our later simulations 
we restrict ourselves to $k_{\text {diss }}=0$ so as to be consistent with other models that disregard the effect of C-S-H dissolution.

The hydration chemistry of other cement constituents such as aluminates, ferrites, etc. are not considered here because they do not contribute appreciably to the porous structure of the concrete [13, 12. Instead, we focus on the effect of C-S-H gel on decreasing porosity and hindering moisture transport within the porous concrete matrix.

\section{Mathematical model}

We begin by providing a list of primary simplifying assumptions that will permit us to reduce the complexity of the governing equations while at the same time retaining the essential aspects of the underlying physical and chemical processes:

1. The length scales under consideration are large enough that the solid concrete matrix can be treated as a continuum. Consequently, volume fractions and constituent concentrations can be expressed as continuous functions of space and time, and the liquid transport is assumed to obey Darcy's law.

2. The concrete sample is long and thin so that transport can be assumed to be one-dimensional. This is consistent with many experiments involving concrete or other building materials 3 in which the sample under study takes the shape of a long cylinder as pictured in Fig. 17.

3. Temperature variations and heat of reaction can be ignored. This is a reasonable assumption in re-wetting of hardened concrete for which the quantities of silicate reactants are much smaller than during the initial hydration stage [26].

4. Water transport is dominated by capillary action and so gravitational effects can be ignored. This assumption is justified by the very small pore dimensions that lead to a small Bond number for concrete [35].

5. We neglect the dynamics of individual ionic species, which is consistent with the work of Bentz [11] and others. Nonetheless, we do consider separate aqueous and solid phases of C-S-H and include a simple dynamic mechanism for precipitation and dissolution which is shown in [46 to be an important effect. This choice is motivated by the recognized complexity of the C-S-H precipitation/crystallization process 22, that is largely ignored by other models of hydration.

6. The effects of chemical shrinkage and subsequent self-desiccation can be significant during initial cement hydration, particularly for high performance concrete [40]. However, we neglect both effects since the samples under consideration are normal strength concrete, and residual silicate concentrations are much smaller during re-wetting. 
7. Reaction kinetics take a simple form in which the rate has a power-law dependence on reactant concentration - a common assumption employed in other models [38, 37.

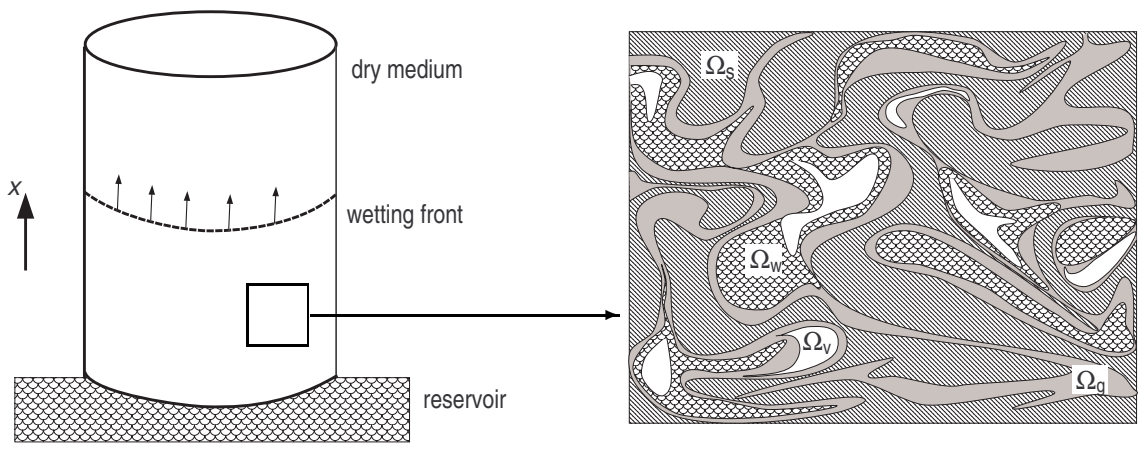

a. Cylindrical concrete sample, depicting the location of a typical wetting front. b. A zoomed-in view at the pore scale, showing a representative elementary volume $\Omega$.

Figure 1: Geometry of the 1D moisture transport problem.

\subsection{Definition of volume fractions and concentrations}

Consider an elementary volume $\Omega\left[\mathrm{cm}^{3}\right]$ pictured in Fig. 1 $1 \mathrm{~b}$ which is divided into sub-volumes occupied by the various components of the porous matrix, namely the non-gel solids with volume $\Omega_{s}$, the precipitated C-S-H gel $\Omega_{g}$, liquid water $\Omega_{w}$, and gas/vapour component $\Omega_{v}$. The pore volume available for transport is denoted by $\Omega_{p}=\Omega_{w}+\Omega_{v}$ and so the total volume can be written as

$$
\Omega=\Omega_{s}+\Omega_{g}+\Omega_{p}=\Omega_{s}+\Omega_{g}+\Omega_{w}+\Omega_{v}
$$

We next define the various volume fractions beginning with the pore volume fraction $\varepsilon=\Omega_{p} / \Omega\left[\mathrm{cm}^{3} / \mathrm{cm}^{3}\right]$, which is also known as porosity. The initial porosity in the absence of C-S-H is denoted by the constant $\varepsilon^{o}=\left.\varepsilon\right|_{t=0}=\left(\Omega-\Omega_{s}\right) / \Omega$. The gel volume fraction is $\varepsilon_{g}=\Omega_{g} / \Omega$ and volumetric water content is $\theta=\Omega_{w} / \Omega$. In practice, $\theta$ must satisfy $0 \leq \theta_{\min } \leq \theta \leq \theta_{\max } \leq \varepsilon^{o}$, where $\theta_{\min }$ is the immobile or residual water content and $\theta_{\max }$ is the maximum or fully saturated value (representing the point beyond which water can no longer penetrate the smallest pores). In the context of cement hydration, there are three forms of water present: chemically bound, physically bound, and capillary water. The quantity $\theta_{\min }$ corresponds to both physically bound (absorbed) and capillary water, which together represent the "evaporable water" that can be removed only by forced drying. All volume fractions will in general be functions of both position and time owing to variations in the gel, liquid, and gas concentrations. 
We next define the concentrations of the various constituents (in units of $\left.\left[\mathrm{g} / \mathrm{cm}^{3}\right]\right)$, which are measured relative to the total mass of concrete following Ref. [38]:

$$
\begin{array}{ll}
C_{\alpha}(x, t)- & \text { concentration of } \mathrm{C}_{3} \mathrm{~S} \text { in concrete, } \\
C_{\beta}(x, t)- & \text { concentration of } \mathrm{C}_{2} \mathrm{~S} \text { in concrete, } \\
C_{q}(x, t)- & \text { concentration of C-S-H in liquid, } \\
C_{g}(x, t)- & \text { concentration of solid C-S-H gel }=\rho_{g} \Omega_{g} / \Omega=\rho_{g} \varepsilon_{g} .
\end{array}
$$

All solution components are taken to be functions of time $t$ and axial distance $x$, where $t \geq 0$ and $0 \leq x \leq L$. The gel-modified porosity $\varepsilon(x, t)$ is related to C-S-H gel concentration via

$$
\varepsilon=\frac{\Omega-\Omega_{s}-\Omega_{g}}{\Omega}=\frac{\Omega-\Omega_{s}}{\Omega}-\frac{\Omega_{g}}{\Omega}=\varepsilon^{o}-\frac{C_{g}}{\rho_{g}},
$$

where $\rho_{g}$ is the density of C-S-H in gel form $\left[\mathrm{g} / \mathrm{cm}^{3}\right]$.

\subsection{Derivation of the governing equations}

We next derive the differential equations governing the water content $\theta$ and each of the constituent concentrations $C_{\alpha}, C_{\beta}, C_{q}$ and $C_{g}$. Conservation of liquid in the pores requires that

$$
\frac{\partial \theta}{\partial t}=-\frac{\partial u}{\partial x}-\nu\left(\theta-\theta_{r}\right)+\frac{m_{w} r_{c s h}}{\rho_{w} m_{c s h}}
$$

where $u$ is the water velocity $[\mathrm{cm} / d a y]$ and $\rho_{w}$ is its density $\left[\mathrm{g} / \mathrm{cm}^{3}\right]$. The reaction term is scaled by a factor $\left(\theta-\theta_{r}\right)^{+}=\max \left(\theta-\theta_{r}, 0\right)$ which reflects the assumption that reactions proceed only when water content is above some minimum value $\theta_{r}$. A similar approach was used in studies of concrete carbonation [37, 42] wherein the value of $\theta_{r}$ is obtained experimentally; in the absence of experimental data for hydration, we simply take $\theta_{r}=\theta_{\text {min }}$. The expression, $r_{c s h}$ representing the rate of generation of C-S-H $\left[\mathrm{g} / \mathrm{cm}^{3}\right.$ day $]$ must be scaled here by the ratio of the molar masses of water and C-S-H, $m_{w} / m_{c s h}$. We also multiply the reaction term by a stoichiometric coefficient $\nu$, which is taken equal to 5 so as to balance the rate of generation of water with the averaged rate coefficient for C-S-H coming from Eqs. (11) and (2). This and other reaction terms are specified later in Section 3.4 .

We assume the liquid velocity can be expressed as

$$
u=-D(\theta, \varepsilon) \frac{\partial \theta}{\partial x}
$$

which follows from a simple application of Darcy's law $*$, where the effective diffusivity $D(\theta, \varepsilon)\left[\mathrm{cm}^{2} /\right.$ day $]$ is a function of both water content and porosity

*Darcy's law states that the velocity $u=K \partial h / \partial x$, where $h$ is the pressure head and $K$ is the hydraulic conductivity of the medium. Conductivity is known to depend on porosity, and for variably saturated media both $K$ and $h$ are typically taken to be functions of saturation, such as in the van Genuchten or Brooks-Corey models [7]. Therefore, Darcy's law takes the form of Eq. (6) with $D=K(\theta, \varepsilon) d h / d \theta$. 
(for which a specific functional form will be presented in Section 3.5). After substituting (6) into (5), we obtain

$$
\frac{\partial \theta}{\partial t}=\frac{\partial}{\partial x}\left[D(\theta, \varepsilon) \frac{\partial \theta}{\partial x}\right]-\nu\left(\theta-\theta_{r}\right)+\frac{m_{w} r_{c s h}}{\rho_{w} m_{c s h}} .
$$

Dissolved alite and belite are advected with the pore liquid as well as being affected by diffusion and reaction, and so the corresponding conservation equations are

$$
\begin{aligned}
& \frac{\partial\left(\theta C_{\alpha}\right)}{\partial t}=\frac{\partial}{\partial x}\left(\theta D_{\alpha} \frac{\partial C_{\alpha}}{\partial x}\right)-\frac{\partial\left(u C_{\alpha}\right)}{\partial x}-\left(\theta-\theta_{r}\right)^{+} r_{\alpha}, \\
& \frac{\partial\left(\theta C_{\beta}\right)}{\partial t}=\frac{\partial}{\partial x}\left(\theta D_{\beta} \frac{\partial C_{\beta}}{\partial x}\right)-\frac{\partial\left(u C_{\beta}\right)}{\partial x}-\left(\theta-\theta_{r}\right)^{+} r_{\beta},
\end{aligned}
$$

where $D_{j}, j=\alpha, \beta$ is the diffusivity of each dissolved constituent. Transport of aqueous $\mathrm{C}-\mathrm{S}-\mathrm{H}$ is governed by

$$
\begin{aligned}
\frac{\partial\left(\theta C_{q}\right)}{\partial t}= & \frac{\partial}{\partial x}\left(\theta D_{q} \frac{\partial C_{q}}{\partial x}\right)-\frac{\partial\left(u C_{q}\right)}{\partial x} \\
& +\left(\theta-\theta_{r}\right)^{+}\left(r_{c s h}-k_{\text {prec }} C_{q}+k_{\text {diss }} C_{g}\right)
\end{aligned}
$$

where $k_{\text {prec }}$ and $k_{\text {diss }}$ are the rates of C-S-H precipitation and dissolution respectively. The solid C-S-H phase is not affected by advective or diffusive transport and so obeys a simple ODE

$$
\frac{\partial\left(\theta C_{g}\right)}{\partial t}=\left(\theta-\theta_{r}\right)^{+}\left(k_{p r e c} C_{q}-k_{d i s s} C_{g}\right) .
$$

In summary, the governing equations consist of (77), (8)-(11), which enforce conservation of water, aqueous species, and solid C-S-H gel, supplemented with the relationships (4) and (6).

\subsubsection{Analogy with bioremediation models}

Before presenting the remaining details, it is worthwhile mentioning that there is a great deal of similarity between our model for reactive transport in concrete and those developed for biofilm growth and bioremediation in the soil sciences literature (for example, 16, 15, 29. In the case of bioremediation, bacteria are employed in porous aquifers in order to break down some targeted contaminant. Nutrients (typically nitrates) are injected into the ground to activate the decontamination process and soil scientists are interested in understanding how to encourage the growth of the bacteria in a controlled manner so as to avoid clogging the pores in the rock or soil matrix while at the same time maximizing the breakdown of contaminant. The governing equations for both problems therefore have a similar structure, with a few key differences that we summarize below: 
- In biofilms water is an inert phase, whereas in concrete it participates in the reaction.

- Biological organisms are typically modelled using Monod reaction terms, whereas we use power-law kinetics.

- Biofilms are composed of living cells and so give rise to additional terms that encompass cell division and death processes.

- The microstructure of biofilms and C-S-H are quite different, but our use of a continuum approach means that we can ignore such details. We do nonetheless employ the same power-law form (20) of the permeability correction as that used in biofilms.

\subsection{Initial and boundary conditions}

We assume that the concrete sample at the beginning of an experiment is homogeneous in composition and uniformly hydrated so that the initial water content and concentrations for $0<x<L$ are

$$
\begin{gathered}
\theta(x, 0)=\theta_{\text {min }}, \quad C_{\alpha}(x, 0)=C_{\alpha}^{o}, \quad C_{\beta}(x, 0)=C_{\beta}^{o}, \\
C_{q}(x, 0)=C_{q}^{o}, \quad C_{g}(x, 0)=C_{g}^{o},
\end{gathered}
$$

where the zero superscript denotes a constant initial value. The first condition on water content states that the concrete is initially at the minimum value, corresponding for example to a sample that is equilibrated in a humidified environment but not force-dried. It is reasonable to take the initial C-S-H concentrations $C_{q}^{o}=C_{g}^{o}=0$, but the alite and belite concentrations are key model parameters that depend on the composition of the initial cement mixture. In particular, Papadakis et al. 38] calculate the initial concentrations as

$$
C_{j}^{o}=\left(1-f_{j}\right) \omega_{j} C_{m i x}
$$

where

$$
C_{m i x}=\frac{\rho_{c e m}}{R_{w / c} \rho_{c e m} / \rho_{w}+R_{a / c} \rho_{c e m} / \rho_{a g g}+1}
$$

represents the initial concentration of cement before onset of hydration, $\rho_{c e m}$ is the original cement density, $\rho_{a g g}$ is the particle density of aggregates, $R_{w / c}$ and $R_{a / c}$ are initial water-to-cement and aggregate-to-cement ratios by mass, and $\omega_{j}$ is the mass fraction for each constituent $j=\alpha, \beta$. We have modified Papadakis et al.'s formula slightly to include the extra factors $\left(1-f_{j}\right)$ where $f_{j} \in[0,1]$ represents the fractional degree of hydration of each constituent at the end of the hydration/curing stages.

The cement mixtures investigated in [3] contain significant levels of tricalcium aluminate (or $\mathrm{C}_{3} \mathrm{~A}$, short for $3 \mathrm{CaO} \cdot \mathrm{Al}_{2} \mathrm{O}_{3}$ ) and no tetracalcium aluminoferrite. Consequently, for the purposes of calculating initial porosity, we also 
include the effect of $\mathrm{C}_{3} \mathrm{~A}$, whose initial hydration products further reduce the pore space available for transport. Letting $f_{\gamma}$ and $\omega_{\gamma}$ refer to the mass and hydration fractions for $\mathrm{C}_{3} \mathrm{~A}$, we are led to the following expression for initial porosity [38, Tab. 2]:

$$
\varepsilon^{o}=C_{m i x} R_{w / c} / \rho_{w}-C_{m i x}\left(f_{\alpha} \omega_{\alpha} \Delta V_{\alpha}+f_{\beta} \omega_{\beta} \Delta V_{\beta}+f_{\gamma} \omega_{\gamma} \Delta V_{\gamma}\right),
$$

where the first term represents the porosity before onset of hydration and the remaining terms encompass the reduction in pore volume owing to hydration through parameters $\Delta V_{\alpha}=0.233, \Delta V_{\beta}=0.228$ and $\Delta V_{\gamma}=0.555$ (units of $\left.\left[\mathrm{cm}^{3} / \mathrm{g}\right]\right)$.

We note in passing that the strength of the resulting hardened concrete is related to $R_{w / c}$ and $R_{a / c}$ as well as the curing conditions. For example, a high value of $R_{w / c}$ yields a low strength concrete owing to an increase in porosity that occurs because of the excess water present during hydration; consequently, most concrete is mixed with an initial water-to-cement ratio ranging from 0.30 to 0.60 .

The bottom end of the concrete sample is immersed in water, where we impose the following Dirichlet boundary condition

$$
\theta(0, t)=\theta_{\max }-\frac{C_{g}(0, t)}{\rho_{g}},
$$

which states simply that the sample is fully saturated at $x=0$. We also assume perfect sink conditions on the aqueous species, so that

$$
C_{j}(0, t)=0 \quad \text { for } j=\alpha, \beta, q \text {. }
$$

In typical experiments, the concrete sample is coated on the sides and top face with a sealant (such as epoxy) that prevents any transport into or out of the sample. This supports our 1D approximation and allows us to impose homogeneous Neumann boundary conditions

$$
\frac{\partial \theta}{\partial x}(L, t)=0 \quad \text { and } \quad \frac{\partial C_{j}}{\partial x}(L, t)=0,
$$

where $j=\alpha, \beta, q$. These conditions are equivalent to imposing a zero flux because the boundary condition on $\theta$ at $x=L$ requires that the convective flux component is zero. We note in closing that no boundary conditions are needed for $C_{g}$ because it is governed by an ODE.

\subsection{Reaction rates}

The reaction terms are specified using notation introduced by Papadakis et al. 38 wherein the rate of generation $r_{j}\left[\mathrm{~g} / \mathrm{cm}^{3} d a y\right]$ of species $j=\alpha, \beta$ is

$$
r_{j}=k_{j} C_{j}\left(\frac{C_{j}}{C_{j}^{o}}\right)^{n_{j}-1},
$$


with $k_{j}\left[d a y^{-1}\right]$ a rate constant, $n_{j}$ a power-law exponent, and $C_{j}^{o}$ the initial concentration (all given in Table 1). The total rate of generation of C-S-H due to the alite and belite reactions $\left[\mathrm{g} / \mathrm{cm}^{3} \mathrm{day}\right]$ is

$$
r_{c s h}=\frac{m_{c s h}}{2}\left(\frac{r_{\alpha}}{m_{\alpha}}+\frac{r_{\beta}}{m_{\beta}}\right),
$$

where $m_{\alpha}, m_{\beta}$ and $m_{c s h}$ are molar masses of alite, belite and C-S-H respectively. A power-law reaction mechanism similar to (18) has also been employed in other models of cement chemistry [37, 42, 17].

\subsection{Moisture diffusion coefficient}

Following the approach used for biofilms in [16] we take the effective diffusivity to be a separable function of the form

$$
D(\theta, \varepsilon)=\varphi^{19 / 6} D^{*}(\theta)
$$

where the influence of porosity on clogging appears as a power law in the quantity $\varphi=\frac{\varepsilon-\theta_{\min }}{\varepsilon^{o}-\theta_{\min }}$. Clement et al. initially assume that the ratio of porosities obeys $\varphi=\left(R / R_{o}\right)^{m}$, where $R$ and $R_{o}$ represent the corresponding pore radii and $m$ is an empirical constant. They then take two very common functional forms of the constitutive laws for porous media (namely, the van Genuchten and Brooks-Corey relationships) and show that the hydraulic conductivity in both cases satisfies $K / K_{O}=\varphi^{(5 m+4) / 2 m}$; the diffusivity must obey a similar relationship since it is proportional to $K$. By comparing with experimental data from a wide range of soils, Clement et al. find their power-law fit to be insensitive to the specific choice of $m$. They conclude that $m=3$ is a reasonable approximation, which corresponds to the exponent $19 / 6$ used above.

The question remains whether these relationships applied successfully to biofilm growth in soils are also applicable to C-S-H gel formation in concrete. It is certainly true that the physics governing the two processes are very different. Nonetheless, models for formation of C-S-H are based on the premise that the gel precipitates as outgrowths from the surface of cement grains [14] which is analogous to the manner in which biofilms accumulate on soil particles. Furthermore, the derivation above uses only spatially averaged quantities and hence makes no assumption about any specific pattern of biofilm growth. We therefore conclude that the $19 / 6$ rule should also be applicable to concrete.

Turning now to $D^{*}(\theta)$, we observe that many other studies of water transport in concrete and related porous media [5, 25, 39, 36 approximate the diffusivity by an exponential function of saturation

$$
D^{*}(\theta)=A e^{B \theta},
$$

where parameters $A\left[\mathrm{~cm}^{2} / d a y\right]$ and $B$ are empirical constants. Lockington et al. 34 performed extensive experiments which showed that a number of building materials may be characterized by a universal exponent represented by 
the rescaled parameter $\bar{B}=B\left(\theta_{\max }-\theta_{\min }\right)$ whose value lies between 4 and 6 ; other work [39] suggests that $\bar{B}$ could be as low as 2 and as high as 8 . Note that these parameters lead to very rapid variations in diffusivity over the physical range of saturations (by at least three orders of magnitude) which distinguishes water transport in concrete from that of many other common porous media.

\subsection{Choice of base case parameters}

The numerical simulations in this paper focus on reproducing experimental results reported by Barrita et al. [4, 3] and specifically the concrete sample they refer to as "mixture 3." We begin by selecting a representative set of parameters for a "base case" simulation, but since not all of the required data is provided in these references we have had to estimate certain values using other literature sources. The parameters are summarized in Table 1 and we comment below on a number of the more critical choices:

Sample geometry. We have taken the model domain to have length $L=$ $10 \mathrm{~cm}$ which is consistent with the cylindrical samples of concrete used in [3].

Water transport coefficients. The maximum water content is $\theta_{\max }=0.067$, which is equal to the initial porosity for the base case and is consistent with measured values reported in the concrete literature [5, 31. The residual water content is taken to be a small positive number because concrete is typically not totally free of water unless it has been artificially dried [19] and in practice some small amount of water is typically trapped within the porous concrete matrix; specifically, we choose a value of $\theta_{\min }=0.04$ by estimating the minimum water content from plots in 44. We take the diffusion parameter $B=100$, which is chosen so that the rescaled quantity $\bar{B}=B\left(\theta_{\max }-\theta_{\min }\right)=2.66$ (for the base case and other simulations performed later) is consistent with the range of values mentioned in Section 3.5. The value of $A=0.0028$ then follows by fitting the simulated wetting curves to Barrita's experimental results (more details are provided in Section 4.1).

Diffusion coefficients for aqueous species. Since the alite and belite actually dissociate and diffuse as ions, the best we can do is to use an approximation that represents the diffusivities in some averaged sense. We begin with the diffusivities of the ionic constituents $\mathrm{Ca}^{2+}, \mathrm{OH}^{-}$and $\mathrm{H}_{2} \mathrm{SiO}_{4}^{2-}$, which are equal to $0.68,4.6$, and $0.43 \mathrm{~cm}^{2} / d$ respectively [46], and compute an appropriately-scaled harmonic average of approximately $1.0 \mathrm{~cm}^{2} / d$ for both alite and belite (following the development in [18). The diffusion of ions in cementitious materials is known to be reduced by a factor ranging from $10^{-1}$ to $10^{-3}[20$, that depends on the pore structure and cement composition; in the absence of any better information we choose a factor of $10^{-2}$ after which $D_{\alpha}=D_{\beta}=0.01 \mathrm{~cm}^{2} / d$. The C-S-H gel does not diffuse in ionic form, and since no data is available in the literature regarding its diffusion coefficient we have chosen to simply take the same value $D_{q}=0.01 \mathrm{~cm}^{2} / d$. This is not so much of a concern, since we investigate later on in Section 4.6 the effect of varying $D_{j}$ and demonstrate that 
Table 1: Parameter values corresponding to the base case.

\begin{tabular}{|c|c|c|c|c|}
\hline Symbol & Description & Value & Units & Reference \\
\hline$\rho_{w}$ & Liquid water density & 1.0 & $\mathrm{~g} / \mathrm{cm}^{3}$ & \\
\hline$\rho_{g}$ & C-S-H gel density & 2.6 & $\mathrm{~g} / \mathrm{cm}^{3}$ & Allen et al. 2] \\
\hline$\rho_{\text {cem }}$ & Cement density & 2.83 & $\mathrm{~g} / \mathrm{cm}^{3}$ & Barrita et al. 3 \\
\hline$\rho_{\text {agg }}$ & Aggregate particle density & 2.6 & $\mathrm{~g} / \mathrm{cm}^{3}$ & \\
\hline$m_{\alpha}$ & Alite molar mass & 228.3 & $\mathrm{~g} / \mathrm{mol}$ & \\
\hline$m_{\beta}$ & Belite molar mass & 172.2 & $\mathrm{~g} / \mathrm{mol}$ & \\
\hline$m_{w}$ & Water molar mass & 18.0 & $\mathrm{~g} / \mathrm{mol}$ & \\
\hline$m_{c s h}$ & C-S-H molar mass & 342.4 & $\mathrm{~g} / \mathrm{mol}$ & \\
\hline$D_{\alpha}$ & Alite diffusivity & 0.01 & $\mathrm{~cm}^{2} / \mathrm{day}$ & \\
\hline$D_{\beta}$ & Belite diffusivity & 0.01 & $\mathrm{~cm}^{2} / \mathrm{day}$ & \\
\hline$D_{q}$ & C-S-H (aq) diffusivity & 0.01 & $\mathrm{~cm}^{2} / \mathrm{day}$ & \\
\hline$A^{4}$ & Water diffusion coefficient & 0.0028 & $\mathrm{~cm}^{2} / d a y$ & \\
\hline$B$ & Water diffusion exponent & 100 & - & Lockington et al. 34] \\
\hline$\theta_{\min }$ & Residual water content & 0.04 & - & Barrita 4] \\
\hline$\theta_{r}$ & Reaction cut-off & 0.04 & - & Equal to $\theta_{\min }$ \\
\hline$k_{\alpha}$ & Alite reaction rate & 22.2 & $d a y^{-1}$ & Papadakis et al. 38 \\
\hline$k_{\beta}$ & Belite reaction rate & 3.04 & $d a y^{-1}$ & " \\
\hline$n_{\alpha}$ & Alite reaction exponent & 2.65 & - & $"$ \\
\hline$n_{\beta}$ & Belite reaction exponent & 3.10 & - & $"$ \\
\hline$k_{\text {prec }}$ & C-S-H precipitation rate & 32.2 & $d a y^{-1}$ & Bentz [12] \\
\hline$k_{\text {diss }}$ & C-S-H dissolution rate & 0 & $d a y^{-1}$ & $"$ \\
\hline$\nu$ & Water stoichiometry & 5 & - & Eqs. (11) and (2) \\
\hline$R_{w / c}$ & Water-to-cement ratio & 0.333 & - & Barrita et al. 3 \\
\hline$R_{a / c}$ & Aggregate-to-cement ratio & 2.86 & - & $"$ \\
\hline$\omega_{\alpha}$ & Alite mass fraction & 0.65 & - & $"$ \\
\hline$\omega_{\beta}$ & Belite mass fraction & 0.17 & - & $"$ \\
\hline$\omega_{\gamma}$ & $\mathrm{C}_{3} \mathrm{~A}$ mass fraction & 0.11 & - & $"$ \\
\hline$f_{\alpha}$ & Alite hydration fraction & 0.60 & - & Tennis \& Jennings 44 \\
\hline$f_{\beta}$ & Belite hydration fraction & 0.20 & - & $"$ \\
\hline$f_{\gamma}$ & $\mathrm{C}_{3} \mathrm{~A}$ hydration fraction & 0.72 & - & $"$ \\
\hline$\Delta V_{\alpha}$ & Alite volume change & 0.233 & $\mathrm{~cm}^{3} / \mathrm{g}$ & $"$ \\
\hline$\Delta V_{\beta}$ & Belite volume change & 0.228 & $\mathrm{~cm}^{3} / \mathrm{g}$ & $"$ \\
\hline$\Delta V_{\gamma}$ & $\mathrm{C}_{3} \mathrm{~A}$ volume change & 0.555 & $\mathrm{~cm}^{3} / \mathrm{g}$ & $"$ \\
\hline$L$ & Length of sample & 10.0 & $\mathrm{~cm}$ & Barrita et al. [3] \\
\hline$C_{q}^{o}$ & Initial C-S-H (aq) concentration & 0 & $\mathrm{~g} / \mathrm{cm}^{3}$ & \\
\hline$C_{g}^{o}$ & $\begin{array}{l}\text { Initial C-S-H (gel) concentra- } \\
\text { tion }\end{array}$ & 0 & $\mathrm{~g} / \mathrm{cm}^{3}$ & \\
\hline \multicolumn{5}{|c|}{ Derived parameters: } \\
\hline$C_{\alpha}^{o}$ & Initial alite concentration & 0.145 & $\mathrm{~g} / \mathrm{cm}^{3}$ & Eq. (13) \\
\hline$C_{\beta}^{o}$ & Initial belite concentration & 0.076 & $\mathrm{~g} / \mathrm{cm}^{3}$ & $"$ \\
\hline$\varepsilon^{o}$ & Initial porosity & 0.067 & - & Eq. (14) \\
\hline$\theta_{\max }$ & Maximum water content & 0.067 & - & \\
\hline
\end{tabular}


the solution is relatively insensitive to the values of diffusivity.

$\mathrm{C}-\mathrm{S}-\mathrm{H}$ composition. $\quad \mathrm{C}-\mathrm{S}-\mathrm{H}$ takes on a whole range of possible forms represented by the general formula $\mathrm{C}_{y} \mathrm{~S}_{2} \mathrm{H}_{z}$ and so can only be considered in an averaged sense. We take $m_{c s h}=342.4 \mathrm{~g} / \mathrm{mol}$ as a representative molar mass corresponding to $y=z=3$, which is consistent with many other studies. There is a correspondingly wide range of gel densities reported in the literature, from $1.85 \mathrm{~g} / \mathrm{cm}^{3}$ at the lower end 28] up to $3.42 \mathrm{~g} / \mathrm{cm}^{3}$ [41; we have chosen an intermediate value of $\rho_{g}=2.6 \mathrm{~g} / \mathrm{cm}^{3}$ which is justified by recent work on C-S-H microstructure [2].

Cement composition. According to information provided in $[3]$ on concrete mixture 3 , the mass fractions of silicate constituents in the cement are $\omega_{\alpha}=0.65$, $\omega_{\beta}=0.17$ and $\omega_{\gamma}=0.11$, while the aggregate- and water-to-cement ratios are $R_{a / c}=2.86$ and $R_{w / c}=0.333$. The cement mixture also contains $30 \%$ by weight of fly ash, which is a lower-density pozzolanic additive that improves the strength and workability of the resulting concrete. Based on densities of 3.15 and $2.08 \mathrm{~g} / \mathrm{cm}^{3}$ for Portland cement and fly ash respectively, this translates into an overall cement density of $\rho_{\text {cem }}=2.86 \mathrm{~g} / \mathrm{cm}^{3}$. All concrete samples were moist cured for 7 days which allows us to estimate $f_{\alpha}=0.60, f_{\beta}=0.20$ and $f_{\gamma}=0.72$ from the plot of hydration fractions versus curing time given in [44]. Finally, the aggregates used in all mixtures are a combination of both fine and coarse quartz materials, and so we take $\rho_{\text {agg }}=2.6 \mathrm{~g} / \mathrm{cm}^{3}$ which is representative of the dry particle density of sand.

Alite and belite reaction rates. There is considerable variation in rate parameters reported in the literature owing partly to the fact that many experiments are performed not on cement samples but rather under idealized equilibrium conditions in which reactants are in solution. We have therefore chosen our parameters based on the data provided in 38, who proposed the mechanism (18) along with reaction exponents $n_{\alpha}=2.65$ and $n_{\beta}=3.10$; however if we use their values of $k_{\alpha}=1.01$ and $k_{\beta}=0.138$, then our model exhibits negligible clogging. But in fact, the reaction rate coefficients reported in the literature vary by several orders of magnitude [46, 12, 45, and so this ambiguity has led us to use the reaction rates as fitting parameters. Specifically, we take $k_{\alpha}=22.2$ and $k_{\beta}=3.04$, which lie within the range of published values while also maintaining the same ratio of $k_{\alpha} / k_{\beta}$ used in [38] (more details on the fitting procedure are provided in Section 4.1).

Precipitation and dissolution rates. Bentz [12] developed a model that assumes a linear hydration rate law with rate constant ranging from 0.264 to $1.464 d_{a y}{ }^{-1}$ depending on $R_{w / c}$. We choose the precipitation rate as the upper end of their range, but again scale using the same factor as the other reaction rates to obtain $k_{\text {prec }}=32.2$. We also take $k_{\text {diss }}=0$ following Bentz and others who neglect C-S-H dissolution. 


\section{Numerical simulations}

The governing equations are discretized in space using a centered finite volume approach wherein the domain is divided into $N$ uniform cells having width $h=L / N$ and centered at $x_{i}=(i-1 / 2) h$ for $i=1,2, \ldots, N$. The discrete solution components, for example $C_{i}(t) \approx C_{\alpha}\left(x_{i}, t\right)$, are approximations of the average value of the solution within each cell. Using this notation, the discrete approximation of the alite equation (8) is

$$
\begin{aligned}
\frac{\partial\left(\theta_{i} C_{i}\right)}{\partial t}= & \frac{D_{\alpha}}{h}\left(\theta_{i+1 / 2} \frac{C_{i+1}-C_{i}}{h}-\theta_{i-1 / 2} \frac{C_{i}-C_{i-1}}{h}\right) \\
& -\frac{u_{i+1 / 2} C_{i+1 / 2}-u_{i-1 / 2} C_{i-1 / 2}}{h}-\left(\theta_{i}-\theta_{r}\right)^{+}\left(r_{\alpha}\right)_{i},
\end{aligned}
$$

where the quantities $C_{i \pm 1 / 2}$ are approximations of the solution at the left (-) and right $(+)$ cell edges for which we use an arithmetic average $C_{i \pm 1 / 2}=$ $\left(C_{i}+C_{i \pm 1}\right) / 2$. The discrete velocity at cell edges is written using the centered difference approximation of Darcy's law

$$
u_{i-1 / 2}=-D\left(\theta_{i-1 / 2}, \varepsilon_{, i-1 / 2}\right) \frac{\theta_{i}-\theta_{i-1}}{h} .
$$

The same approach is used to discretize the remaining conservation equations (7), (9), (10) and (11). In all cases, the equations corresponding to boundary cells $i=1$ and $N$ involve "fictitious" solution values located at points $x_{0}=-h / 2$ and $x_{N+1}=L+h / 2$ which lie one-half grid cell outside the domain. The boundary conditions are discretized using second-order differences or averages, and are used to eliminate these fictitious values in terms of interior solution components.

The resulting semi-discretization is fully second order accurate in space and leads to a system of $5 \mathrm{~N}$ ordinary differential equations for the discrete solution values which we then integrate in time using MatLAB's stiff solver ode15s. For all simulations, we use $N=100$ cells and set both relative and absolute error tolerances for ode15s to $10^{-8}$. The equations are integrated to time $t=28$ days, which requires approximately $40 s$ of clock time on a Macintosh PowerBook with a $1.67 \mathrm{GHz}$ PowerPC G4 processor.

\subsection{Base case with and without reactions}

We focus on developing comparisons with the experiments of Barrita [4, 3] who studied wetting of concrete cylinders with both water and isopropanol. The latter solute is particularly useful in such a study because the silicate compounds in concrete do not react with isopropanol as they do with water, and so the isopropanol results may be used to calibrate the diffusion parameter $A$ with experimental data.

In the absence of reactions $\left(k_{\alpha}=k_{\beta}=k_{\text {prec }}=0\right)$ there is no change in constituent concentrations and so the problem reduces to a single nonlinear diffusion equation for the water content. It is well known that for an exponential 
diffusivity of the form (21) with large $B$ and small $A$, the diffusion equation has a solution which forms a steep front that progresses into the sample with speed nearly proportional to the square root of time; consequently, a plot of the isopropanol wetting front location versus $t^{1 / 2}$ should be a straight line, as indicated by the experimental data of Barrita reproduced in Fig. 2 (square points). The experimental results for water uptake also exhibit a linear trend over the first 8-10 hours, which represents a period over which the reactions have not yet begun to take hold and no significant clogging has occurred. However, there is a noticeable difference between the initial slopes of the isopropanol and water data, which is most likely attributable to variations in the concrete samples used, or differences in the capillary pressure or other transport properties for the two liquids. Therefore, we have fit our model to the first 8-10 hours from the water experiment instead of using the isopropanol data.

We proceed by setting $B=100$ and varying $A$ until the slope of the wetting front curve best approximates that of the experimental data. This fitting yields an estimate of $A=0.0028 \mathrm{~cm}^{2} /$ day which is consistent with values reported by other authors such as [1. A plot of the computed wetting front location (without reactions) is displayed in Fig. 2 alongside the corresponding experimental data for comparison purposes. In this and all successive computations, the front location $s(t)$ has been estimated by identifying the point $x$ where the water content comes to within some tolerance of $\theta_{\text {min }}$; that is, $s(t)=\min \{x: \theta(x, t) \leq$ $\left.\theta_{\min }+0.0005\right\}$.

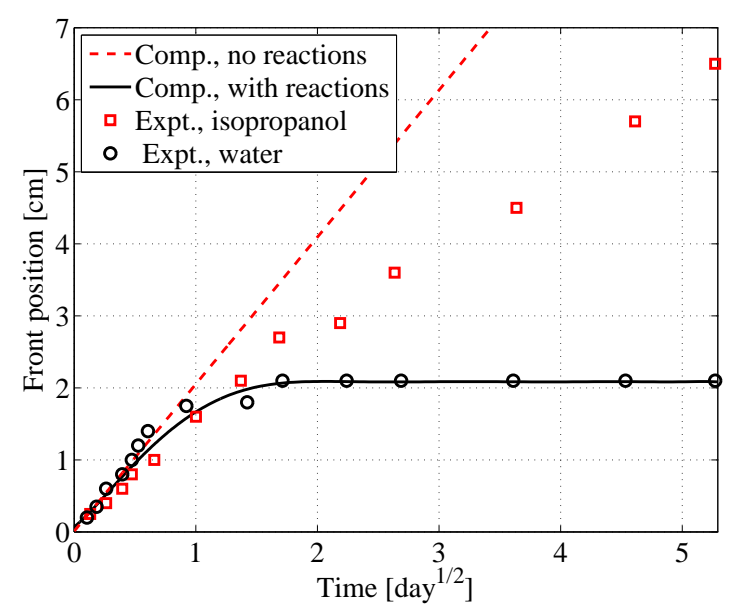

Figure 2: Wetting front location $s(t)$ for the base case computations both with reactions (solid line) and without (dashed line), which should be compared to the experimental data with water (circular points, taken from [4]). The corresponding experimental data for isopropanol (square points) are also included for comparison purposes.

Moving now to the case of water uptake including hydration reactions, it 
remains to choose an appropriate scaling of rate constants in order to best match the location of the stalled wetting front in experiments. As mentioned earlier in Section [3.6, we scale the reaction and absorption rates $k_{\alpha}, k_{\beta}$ and $k_{\text {prec }}$ simultaneously with the same value, while holding their ratio constant at $1.01: 0.138: 1.464$. This procedure yields the rates $k_{\alpha}=22.2, k_{\beta}=3.04$ and $k_{\text {prec }}=32.2$ for which the computed water content profile is displayed for comparison purposes in Fig. 3. We observe that incorporating the effects of hydration reactions and clogging due to $\mathrm{C}-\mathrm{S}-\mathrm{H}$ gel formation clearly causes the wetting front to stall a short distance inside the sample.

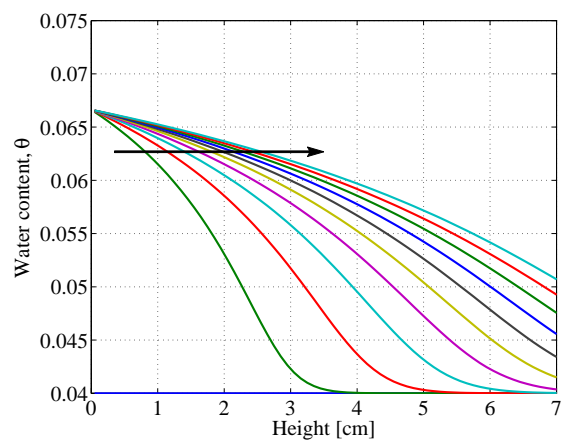

a. Without reactions.

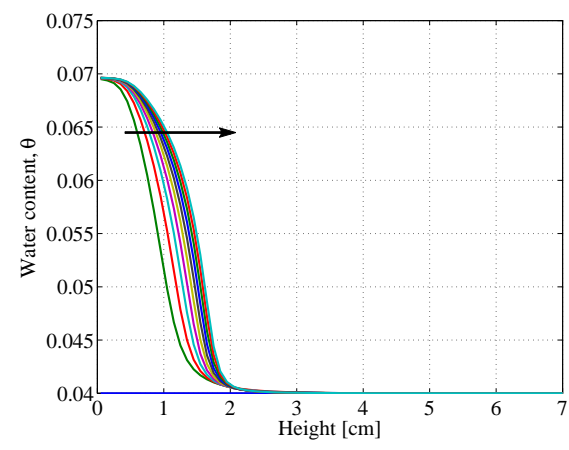

b. With reactions.

Figure 3: Plots of computed water content for the base case parameters, both with and without reactions. The various solution profiles correspond to 10 equally-spaced times over the 28 days of the simulation.

Plots of concentrations and gel-modified porosity are provided in Fig. 4 . which indicate how transport of reactants into the sample is initially dominated by diffusion (for which the front propagates with velocity proportional to $t^{1 / 2}$ ) but then later stalls as C-S-H forms and is precipitated near the lower end of the sample. The onset of clogging can be clearly seen in the gel concentration plots where $C_{g}$ exhibits a peak slightly behind the stall location, while the porosity drops to its minimum value (approx. 0.13) within an interval containing the wetting front and $C_{g}$ peak. It is worthwhile noting that diffusion and reaction processes continue to occur even after the front stalls - most noticeably ahead of the wetting front - owing to the presence of residual pore water, although this process continues at a much slower rate. We emphasize that although the capillary percolation threshold $\theta=\theta_{\text {min }}$ corresponds to the point where water can no longer move by capillary action, there is still sufficient water available for the aqueous components to diffuse (since $\theta$ represents the physically bound or absorbed water as well as capillary water).

The trends shown here suggest that onset of clogging occurs in the interior of the sample to the right of the inflow boundary. This effect can be attributed to a large initial influx of water at $x=0$ that dissolves the alite and belite near the boundary transporting them some distance downstream before the gel 
precipitates. This result is consistent with [4] who reported high values of water flux within the first few hours of their experiments.

We emphasize here that similar stalling behavior has been reported by several other authors performing experiments on porous building materials [43, 32, 35. although these authors attributed this behaviour to an anomalous diffusion mechanism. Our primary aim here has been to show that a similar phenomenon can arise from pore clogging caused by hydration of residual silicates in concrete.

The formation of a wetting front and subsequent stalling due to pore clogging are strongly dependent on two components of our model: the porosity dependence in the diffusion coefficient which drops to zero as $\varepsilon \rightarrow \theta_{\min }$; and the "shut-off" factor $\left(\theta-\theta_{r}\right)^{+}$appearing in the reaction terms. To illustrate the impact of omitting either effect, we present two additional simulations. First, if the porosity correction factor is removed from $D(\theta, \varepsilon)$ in Eq. (20), then the wetting front propagates as if there were no clogging at all. This is evident by comparing the plot of water content in Fig. 5 k with that from the non-reactive case in Fig. 3a . There is clearly no visible effect on the front motion, even though a significant level of C-S-H gel builds up due to the reactions (see Fig. 5b).

To investigate the effect of slightly relaxing the cut-off factor $\left(\theta-\theta_{r}\right)^{+}$in the reaction terms, we replace the zero cut-off with a small positive value of $5 \times 10^{-5}$ when $\theta \leq \theta_{r}$. The wetting front still stalls as indicated in Fig. 6h; however, reactions occur over the entire domain giving rise to a significant concentration of $\mathrm{C}-\mathrm{S}-\mathrm{H}$ gel to the right of the front and a corresponding small reduction in saturation below $\theta_{\text {min }}$. This effect may be attributed to self-desiccation; however, with no more guidance in how to determine the value of the cut-off parameter, we leave the study of this effect as a possible avenue for future work and retain the factor $\left(\theta-\theta_{r}\right)^{+}$as is. Taking a larger value of the cut-off (close to $\theta_{\min }$ in magnitude) can lead to runaway reactions and instabilities in the numerical method.

We conclude from these last two simulations that in order for our model to give a reasonable picture of clogging observed in re-wetting experiments, there must be some retarding of liquid transport through a porosity dependence in the water diffusivity, and furthermore the reactions must include a shut-off term similar to $\left(\theta-\theta_{r}\right)^{+}$, although a small positive reaction rate might be allowed near the residual saturation.

\subsection{Grid refinement study}

To ensure that our numerical simulations are computing a consistent solution that converges with the expected order of accuracy, we performed a grid refinement study. The base case simulation was repeated on successively finer grids with $N=25,50,100,200,400,800$, and the solution on the finest grid is treated as the exact solution. The solution error was estimated using the discrete $\ell_{2}$

norm of the difference in aqueous C-S-H concentrations $\left\|C_{q}^{N}-C_{q}^{\text {finest }}\right\|_{\ell_{2}}$; Any solution component would suffice, but we choose $C_{q}$ because it often displays the greatest variations. The results are summarized in Table 2, and the ratio between successive errors indicates that the solution appears to be converging 


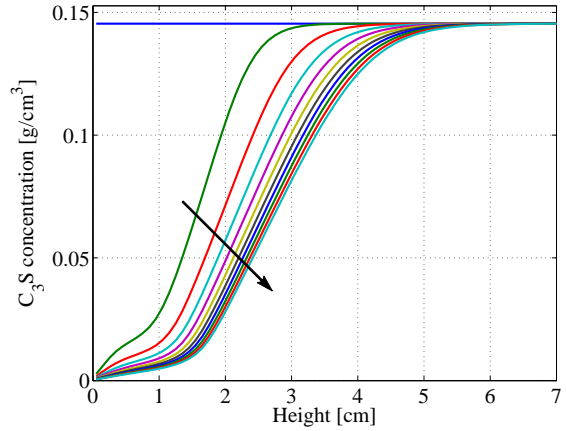

a. Alite concentration, $C_{\alpha}$.

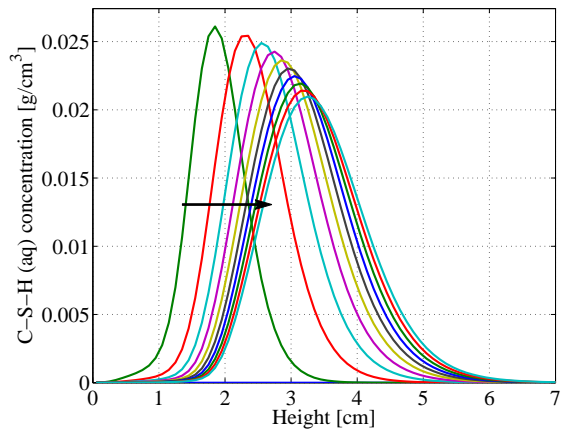

c. Aqueous C-S-H concentration, $C_{q}$.

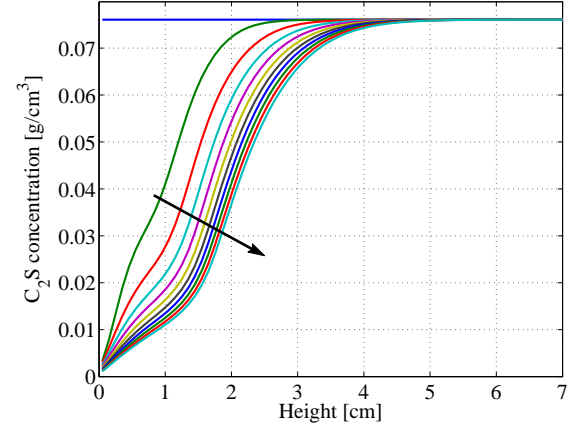

b. Belite concentration, $C_{\beta}$.

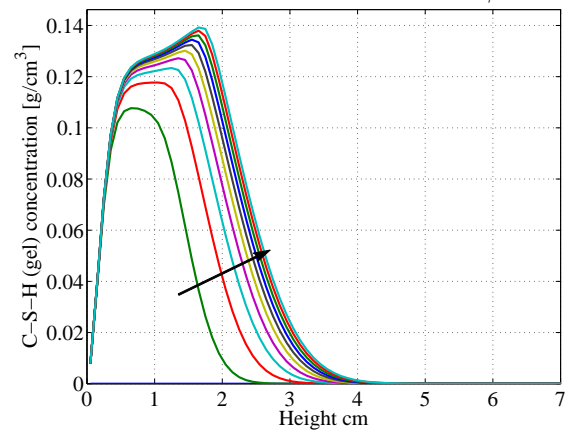

d. C-S-H gel concentration, $C_{g}$.

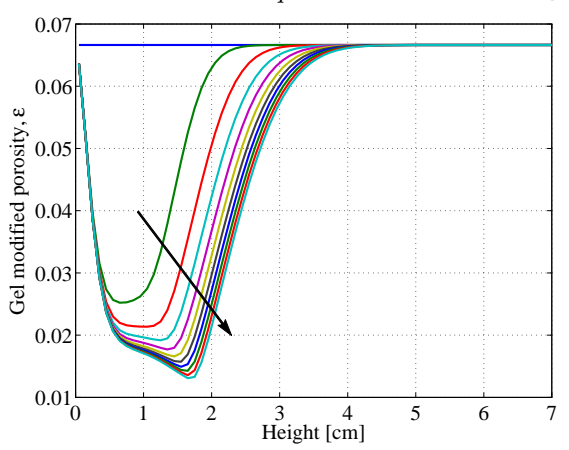

e. Gel modified porosity, $\varepsilon$.

Figure 4: The remaining base case solution profiles corresponding to Figs. 2 and $3 \mathrm{~b}$. In each plot, the solution is displayed at 10 equally-spaced time intervals over 28 days. The arrows on each plot indicate the progression of curves in the direction of increasing time. 


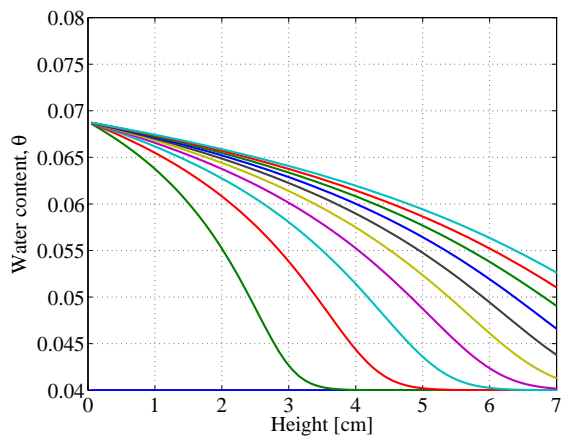

a. Water content.

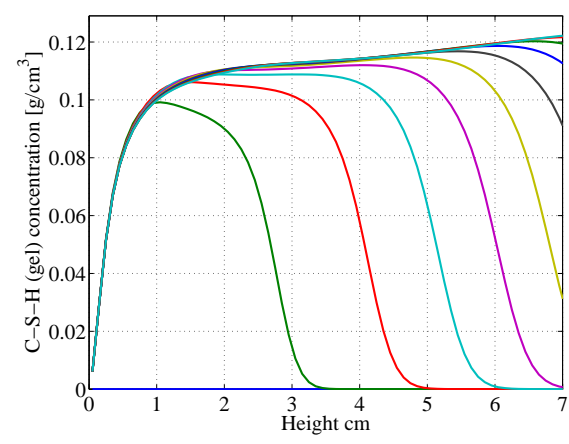

b. C-S-H gel concentration.

Figure 5: Solution with no porosity dependence in the diffusion coefficient, exhibiting an absence of clogging.

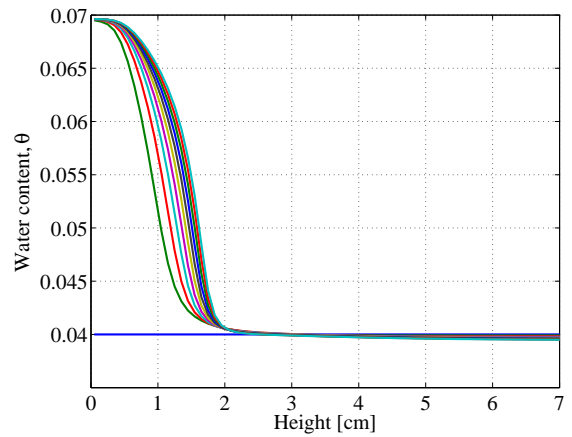

a. Water content.

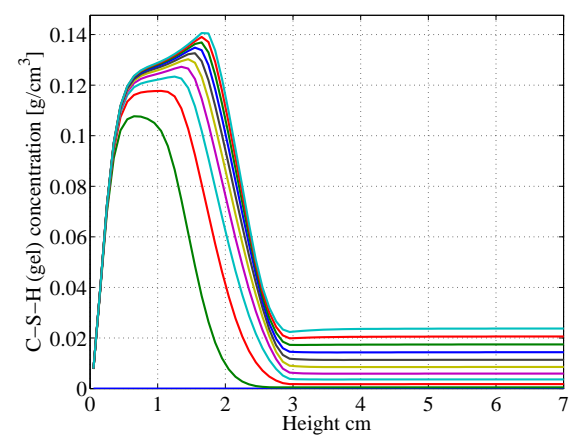

b. C-S-H gel concentration.

Figure 6: Solution computed by replacing $\left(\theta-\theta_{r}\right)^{+} \approx \max \left(\theta-\theta_{r}, 5 \times 10^{-5}\right)$, corresponding to the situation when reactions do not entirely shut off at the residual saturation. 
at a rate that is at least second order, as expected.

Table 2: Grid refinement study. The order is calculated as $\log _{2}$ (ratio).

\begin{tabular}{cccc}
\hline No. of points $(N)$ & $\ell_{2}$-error & Ratio & Order \\
\hline 25 & 0.019 & 2.12 & 1.08 \\
50 & 0.0087 & 4.30 & 2.10 \\
100 & 0.0020 & 5.56 & 2.48 \\
200 & 0.00036 & 6.27 & 2.65 \\
400 & 0.00058 & - & - \\
\hline
\end{tabular}

\subsection{Sensitivity to alite/belite reaction rates}

In this section we vary the reaction rate parameters $k_{\alpha}$ and $k_{\beta}$ to investigate the effect of changes in the individual rates as well as the relative importance of the two reaction routes leading to production of C-S-H gel. To this end we hold $k_{\beta}$ constant and scale $k_{\alpha}$ by the factors $0,0.1$ and 10 , and then repeat the same procedure for $k_{\beta}$. The resulting solutions are displayed in Figs. 7 and 8 from which we see that the clogging seen in the final solution is very sensitive to changes in both rates. The results in both cases are similar, with effect of alite being more pronounced; this is not surprising considering that the initial concentration of alite is significantly larger than that of belite (refer to values of $C_{\alpha}^{o}$ and $C_{\beta}^{o}$ in Table (1). We also note that if the alite reaction rate is taken small enough, then no stalling occurs and the wetting front propagates essentially unhindered into the sample; the same is not true of the belite rate since there is still enough alite being hydrated to cause significant clogging. The sensitivity to reaction rates demonstrated by these results points to the importance of obtaining accurate estimates of the rate parameters.

\subsection{Sensitivity to precipitation rate}

Since there is some uncertainty in the choice of the precipitation rate, it is helpful to consider the effect of changes in $k_{\text {prec }}$. We ran three additional simulations with $k_{\text {prec }}=0.0,3.22$ and 322 and compared those to the base case in Figure 9 . The $k_{\text {prec }}=0$ case is identical to the case displayed in Fig 3a (without reactions) and from the remaining results it is clear that the solution is relatively sensitive to the choice of precipitation rate. We have done our best to choose a value of $k_{\text {prec }}$ consistent with C-S-H precipitation rates in the literature, but there is potentially much to be learned by taking a more detailed look at the precipitation process and including more details about this and other reaction mechanisms in the model equations.

\subsection{Sensitivity to dissolution rate}

We have so far assumed that the formation of C-S-H (gel) is an irreversible process and no dissolution occurs, which is consistent with assumptions made in 


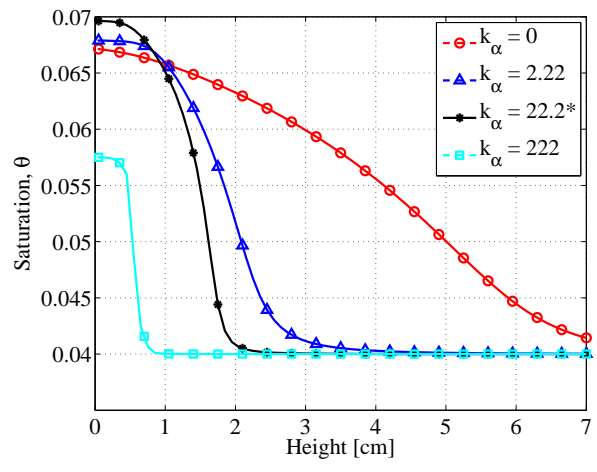

a. Final water content.

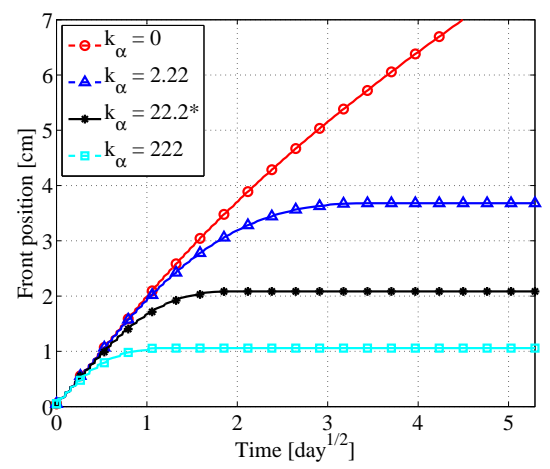

b. Wetting front position.

Figure 7: Water content and wetting front location for different values of the alite reaction rate, $k_{\alpha}$. In this and all succeeding figures, the base case is plotted using a solid black line and highlighted in the legend using "*".

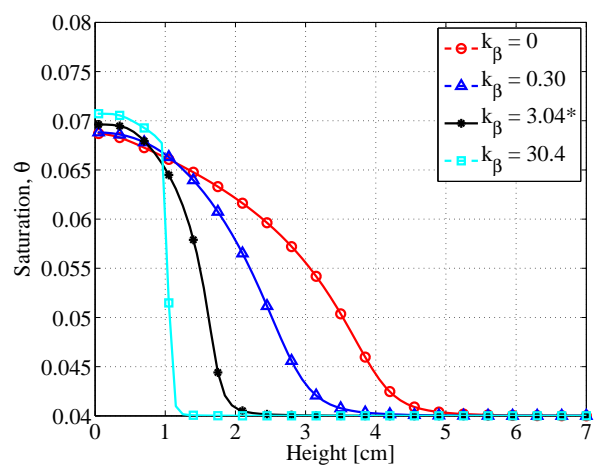

a. Final water content.

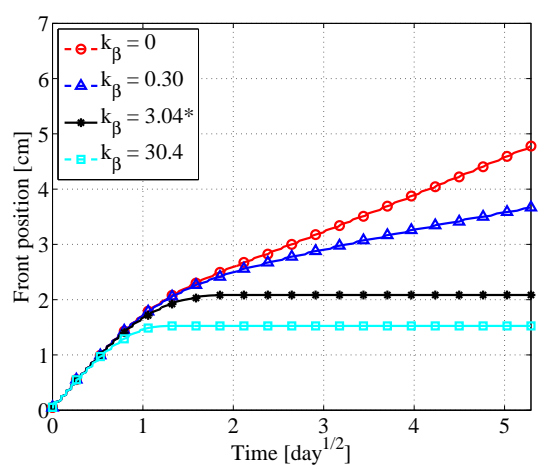

b. Wetting front position.

Figure 8: Water content and wetting front location for different values of the belite reaction rate, $k_{\beta}$. 


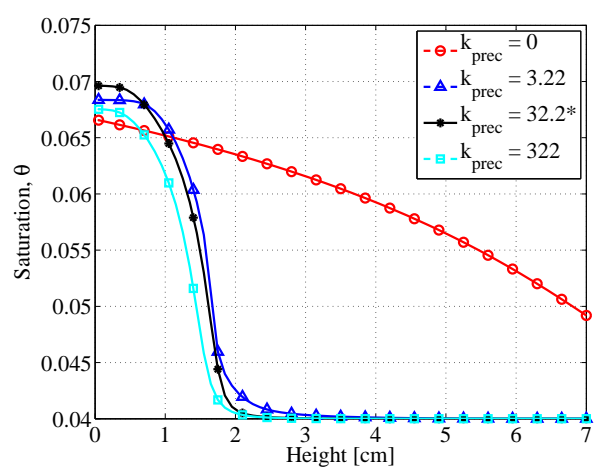

a. Final water content.

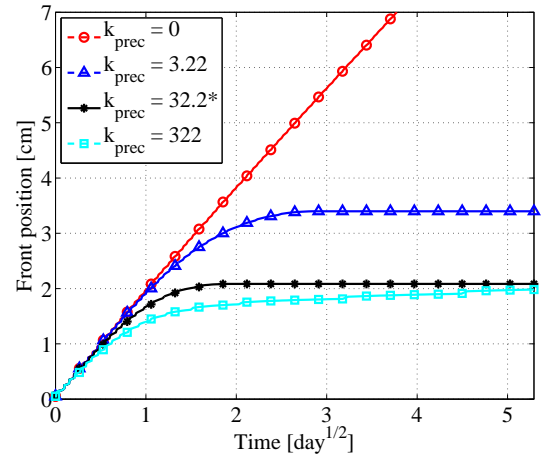

b. Wetting front position.

Figure 9: Water content and wetting front location for different values of the C-S-H precipitation rate, $k_{\text {prec }}$.

many other models. Since our focus is on the phenomenon of re-wetting wherein time scales are much longer than typically considered for initial hydration reactions, it is helpful to consider the effect of incorporating a non-zero dissolution rate constant $k_{\text {diss }}$. To this end, we considered values of $k_{\text {diss }}=1$ and and 10 $d a y^{-1}$ and compared the resulting solutions in Fig. 10, which clearly indicates that only for the largest value of $k_{\text {diss }}$ is there any appreciable effect on the wetting front position, although the water content does show some deviations at smaller values of $k_{\text {diss }}$. These results support our assumption that dissolution has a negligible effect on the solution when $k_{\text {diss }} \ll k_{\text {prec }}$.

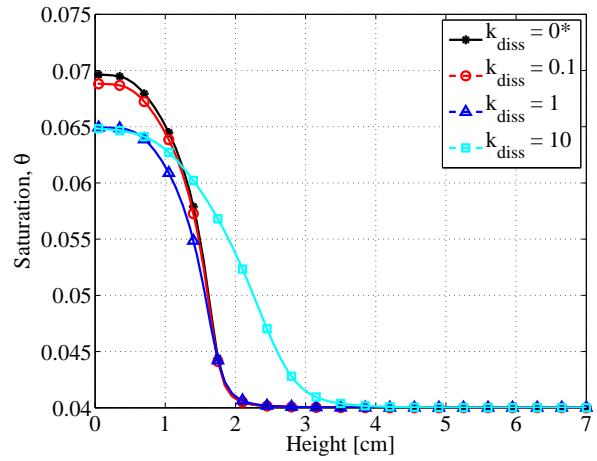

a. Final water content.

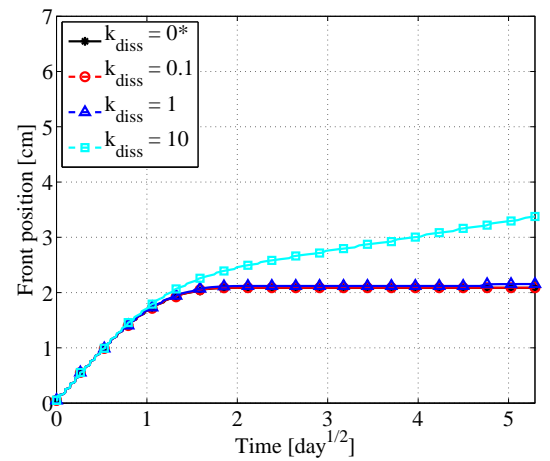

b. Wetting front position.

Figure 10: Water content and wetting front location for different values of the dissolution rate, $k_{\text {diss }}$. 


\subsection{Sensitivity to constituent diffusivity}

We next investigate the effect of changing the diffusion coefficients for the aqueous alite, belite and C-S-H species. We note that our model ignores transport and reaction of individual ionic species and instead approximates the diffusive transport by employing an effective diffusion coefficient for each constituent which may not be entirely representative of how the individual ions would move in response to concentration gradients in solution. Fig. 11 demonstrates that changes in the diffusion coefficient by several orders of magnitude have some effect on the steepness of the wetting front and the distribution of constituents behind it, but have very little influence on the location of the front itself.

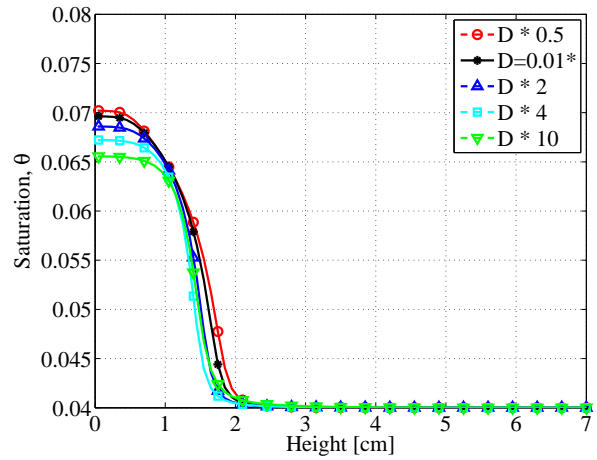

a. Final water content.

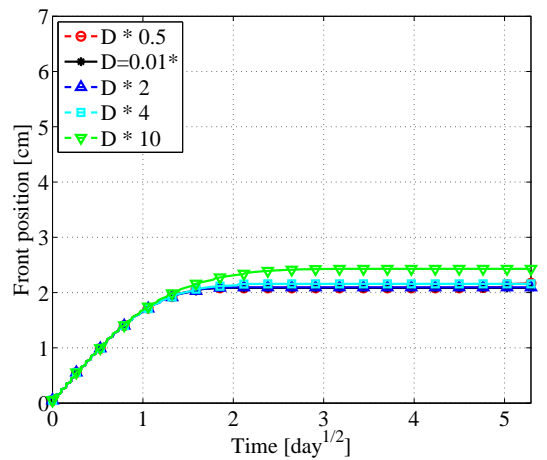

b. Wetting front position.

Figure 11: Water content and wetting front location obtained by varying the diffusivities $D_{\alpha}, D_{\beta}$ and $D_{q}$. In each case depicted, all three diffusivities are scaled by the same constant factor.

\subsection{Sensitivity to aggregate density}

The aggregate materials typically used in concrete include sand and gravel of varying coarseness, all of which have different density. In practice, a combination of various aggregates is frequently used and so we next investigate the effect of variations in the aggregate density. Fig. 12 compares the solution when $\rho_{\text {agg }}$ is varied between 2.4 and 2.8, and shows that even such seemingly small changes in aggregate density can have a measurable effect on clogging; in particular, as $\rho_{\text {agg }}$ increases, the degree of clogging experienced decreases. We therefore conclude that an inaccurate value of the aggregate density parameter could lead to incorrect results.

\subsection{Effect of changes in cement mixture}

Most concrete is mixed with a water-to-cement ratio $R_{w / c}$ lying somewhere between 0.3 and 0.6 . It is well known that when $R_{w / c}$ is too large the resulting 


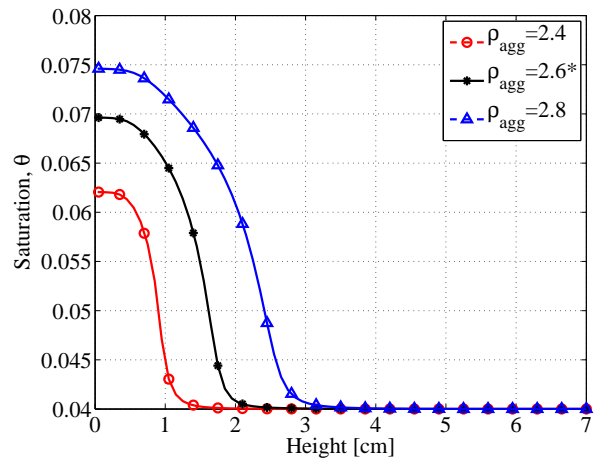

a. Final water content.

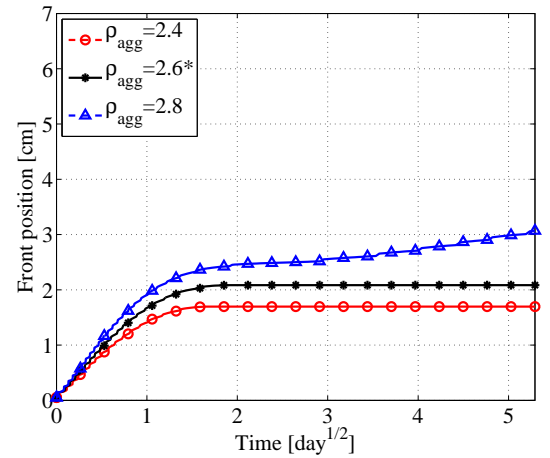

b. Wetting front position.

Figure 12: Water content and wetting front location for different values of the aggregate density, $\rho_{\text {agg }}$.

concrete can be weak and so a smaller $R_{w / c}$ is desirable in general. On the other hand, if there is too little water then the cement can become unworkable or there may even be insufficient pore water to fully hydrate the silicates in the hydration process. Consequently, optimizing concrete strength and durability requires a fine tuning of the initial water content. We have simulated the effect of changes in composition by taking parameters as listed in Table 3. which correspond to mixtures numbered 1 through 4 from [3]. Outside of the variations in $R_{w / c}$ and $R_{a / c}$, a major difference between the various mixtures is the presence of fly ash (in mixtures 2 and 3 ) or silica fume (in mixture 4). Both of these low-density cement additives have the effect of reducing the value of $\rho_{\text {cem }}$, which in the case of mixtures 2 and 3 can change the resulting porosity $\varepsilon^{o}$ significantly.

Table 3: Composition of cement mixtures taken from [3, Tab. 2], with computed results compared in Fig. 13 .

\begin{tabular}{l|cccc}
\hline Mixture & $\rho_{c e m}$ & $R_{w / c}$ & $R_{a / c}$ & $\varepsilon^{o}$ \\
\hline 1 & 3.15 & 0.599 & 5.39 & 0.113 \\
2 & 2.62 & 0.364 & 3.13 & 0.074 \\
3 (base) & 2.83 & 0.333 & 2.86 & 0.066 \\
4 & 3.07 & 0.297 & 3.12 & 0.045 \\
\hline
\end{tabular}

The resulting numerical solutions are compared in Fig. 13 from which it is clear that the initial porosity (as determined by the concrete mixture) can have a major impact on water transport. We note in particular that mixture 1 (with the largest value of $\varepsilon^{o}$ ) exhibits no clogging, while the low value of $\varepsilon^{o}$ in mixture 4 leads to very limited water transport, with the wetting front stalling much closer to $x=0$. Indeed, Barrita et al. 3] observed in experiments that their mixture 4 exhibited a much earlier onset of clogging than the other concrete samples, an effect that is clearly captured in our simulations. However, there 
remains some discrepancy in that experiments on mixture 1 exhibited a stalled wetting front, while our simulations show no clogging in this case.

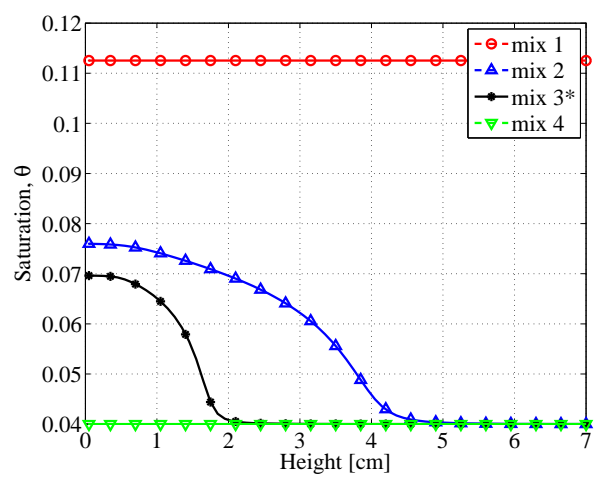

a. Final water content.

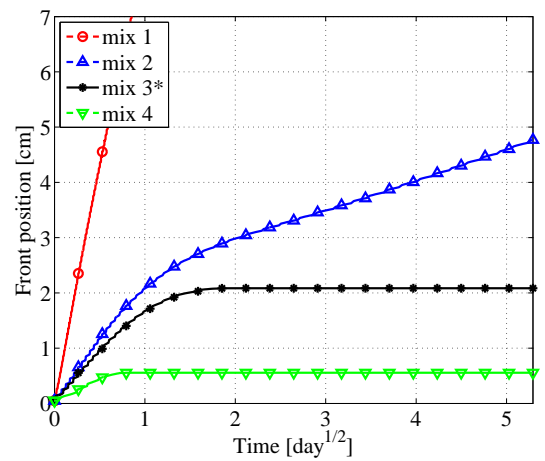

b. Wetting front position.

Figure 13: Water content and wetting front location obtained for various cement compositions, using mixtures 1-4 in [3].

\section{Conclusions and future work}

We have developed a model for the transport and reaction of water and other reactant species in hardened concrete subject to re-wetting. Numerical simulations support our hypothesis that hydration of residual silicates and subsequent formation of C-S-H gel may be responsible for the clogging phenomenon observed in experiments, which is the main contribution of this paper.

We investigated the sensitivity of the solution to changes in a number of model parameters, from which we can conclude that the reaction rate parameters (specifically $k_{\alpha}, k_{\beta}$ and $k_{\text {prec }}$ ) have the most impact on the solution. These are precisely the parameters which are most difficult to ascertain owing to discrepancies in the published literature, and in particular the lack of values for reaction rates in actual concrete as opposed to idealized values obtained for silicates prepared in aqueous solutions. Consequently, more work is required to ensure that inputs to our model are consistent with actual concrete re-wetting scenarios.

In addition to obtaining better estimates of the model parameters, there are a number of extensions to the current model which may significantly improve its predictive power. We expect that the greatest impact may be had by replacing the simple precipitation process embodied in our rate parameter $k_{\text {prec }}$ with a more realistic reaction mechanism that takes into account details of the C-S-H microstructure and hydration which have recently been uncovered. Possible examples include:

- Incorporating the dynamics of individual ionic species through the addi- 
tion of new transport equations and reaction kinetics along the lines of [41] or 37 .

- Investigating the hypothesis put forward in [46 that hydration kinetics is a two-stage process, consisting of an early accelerated hydration step followed by a slower hydration reaction that dominates in the longer term. They suggest that this two-stage kinetics might arise from effects of either C-S-H microstructure or precipitation kinetics, either of which could be considered in detail by appropriate modifications of our model.

- Separating the C-S-H gel into two forms characterized by different densities as suggested in 43, 44, where the lower-density gel is thought to be primarily responsible for changes in porous structure. Taylor et al. 43 also mention the importance of swelling in the cement matrix during initial cement hydration, which is an effect we have so far neglected.

- Chemical shrinkage and the associated phenomenon of self-desiccation, which are known to have a significant impact on initial cement hydration [40].

It may prove useful to incorporate other aspects of porous transport that are commonly seen in modelling studies of ground water aquifers or oil reservoirs, but have yet to be applied to the study of concrete. For example, capillary hysteresis has been identified as an important aspect of cement hydration 8] and results from the soil sciences community [27, 30] could certainly be applied in this context. The issues raised in 21] surrounding the impact of variable porosity on models of multi-phase transport should also be applicable to cement and concrete. Our model can be easily adapted to study other stages in the life of concrete such as initial hydration, carbonation, aging or degradation. Finally, the approach we have developed here would also be applicable to the study of other transport phenomena such as polymer flooding in enhanced oil recovery, where chemical reactions and solution-dependent parameters are important.

\section{Acknowledgements}

This work was supported by grants from the Natural Sciences and Engineering Research Council of Canada and the MITACS Network of Centres of Excellence. JMS was supported by a Research Fellowship from the Alexander von Humboldt Foundation during a visit to the Fraunhofer Institut Techno- und Wirtschaftsmathematik in Kaiserslautern. We thank Dr. Jesús Cano Barrita (Instituto Politécnico Nacional, Oaxaca, Mexico) for many insightful discussions and for providing the experimental data. We are also sincerely grateful to the four anonymous referees whose extensive comments have significantly improved this work. 


\section{References}

[1] H. Akita, T. Fujiwara, and Y. Ozaka. A practical procedure for the analysis of moisture transfer within concrete due to drying. Mag. Concr. Res., 49(179):129-137, 1997.

[2] Andrew J. Allen, Jeffrey J. Thomas, and Hamlin M. Jennings. Composition and density of nanoscale calcium-silicate-hydrate in cement. Nature Mater., 6:311-316, April 2007.

[3] P. F. de J. Cano Barrita, B. J. Balcom, T. W. Bremner, M. B. MacMillan, and W. S. Langley. Moisture distribution in drying ordinary and high performance concrete cured in a simulated hot dry climate. Mater. Struct., 37:522-531, 2004.

[4] Prisciliano Felipe de Jesús Cano Barrita. Curing of high-performance concrete in hot dry climates studied using magnetic resonance imaging. $\mathrm{PhD}$ thesis, University of New Brunswick, Fredericton, NB, November 2002.

[5] B. Bary and A. Sellier. Coupled moisture-carbon dioxide-calcium transfer model for carbonation of concrete. Cement Conc. Res., 34:1859-1872, 2004.

[6] Z. P. Bažant and L. J. Najjar. Drying of concrete as a nonlinear diffusion problem. Cement Conc. Res., 1:461-473, 1971.

[7] Jacob Bear. Dynamics of Fluids in Porous Media. Dover, New York, 1988.

[8] James J. Beaudoin. Why engineers need materials science. Concr. Internat., 21(8):86-89, August 1999.

[9] Arnon Bentur. Cementitious materials - Nine millennia and a new century: Past, present, and future. J. Mater. Civ. Eng., 14(1):2-22, 2002.

[10] D. P. Bentz. Effects of cement PSD on porosity percolation and selfdesiccation. In B. Persson and G. Fagerlund, editors, Self-Desiccation and Its Importance in Concrete Technology II, pages 127-134. Lund University, 1999.

[11] Dale P. Bentz. A three-dimensional cement hydration and microstructure program. I. Hydration rate, heat of hydration and chemical shrinkage. Report No. NISTIR 5756, Building and Fire Research Laboratory, National Institute of Standards and Technology, Gaithersburg, MD, 1995.

[12] Dale P. Bentz. Influence of water-to-cement ratio on hydration kinetics: Simple models based on spatial considerations. Cement Conc. Res., 36(2):238-244, 2006.

[13] J. Billingham, D. T. I. Francis, A. C. King, and A. M. Harrisson. A multiphase model for the early stages of the hydration of retarded oilwell cement. J. Eng. Math., 53:99-112, 2005. 
[14] J. D. Birchall, A. J. Howard, and J. E. Bailey. On the hydration of Portland cement. Proc. Roy. Soc. Lond. A, 360:445-453, 1978.

[15] Michael Chapwanya, Stephen B. G. O'Brien, and J. F. Williams. A 1D bio-clogging model in a phreatic aquifer. In preparation, 2008.

[16] T. P. Clement, B. S. Hooker, and R. S. Skeen. Macroscopic models for predicting changes in saturated porous media properties cause by microbial growth. Ground Water, 34(5):934-942, 1996.

[17] M. M. Y. Delmi, A. Aït-Mokhtar, and O. Amiri. Modelling the coupled evolution of hydration and porosity of cement-based materials. Constr. Build. Mater., 20:504-514, 2006.

[18] Daniels Farrington and Robert A. Alberty. Physical Chemistry. Wiley, 1966.

[19] Daniele Ferretti and Zdeněk P. Bažant. Stability of ancient masonry towers: Moisture diffusion, carbonation and size effect. Cement Conc. Res., 36:1379-1388, 2006.

[20] E. J. Garboczi and D. P. Bentz. Computer simulation of the diffusivity of cement-based materials. J. Mater. Sci., 27:2083-2092, 1992.

[21] W. G. Gray and C. T. Miller. Examination of Darcy's law for flow in porous media with variable porosity. Env. Sci. Tech., 38:5895-5901, 2004.

[22] M. W. Grutzeck. A new model for the formation of calcium silicate hydrate (C-S-H). Mater. Res. Innov., 3:160-170, 1999.

[23] C. Hall, W. D. Hoff, S. C. Taylor, M. A. Wilson, Beom-Gi Yoon, H.-W. Reinhardt, M. Sosoro, P. Meredith, and A. M. Donald. Water anomaly in capillary liquid absorption by cement-based materials. J. Mater. Sci. Lett., 14:1178-1181, 1995.

[24] Christopher Hall. Anomalous diffusion in unsaturated flow: Fact or fiction? Cement Conc. Res., 37:378-385, 2007.

[25] Christopher Hall, W. D. Hoff, and M. Skeldon. The sorptivity of brick: dependence on the initial water content. J. Phys. D, 16:1875-1880, 1983.

[26] Torben C. Hansen. Physical structure of hardened cement paste. A classical approach. Matér. Constr., 19(114):423-436, 1986.

[27] Daniel Hillel. Environmental Soil Physics. Academic Press, 1998.

[28] Hamlin M. Jennings, Jeffrey J. Thomas, Julia S. Gevrenov, Georgios Constantinides, and Franz-Josef Ulm. A multi-technique investigation of the nanoporosity of cement paste. Cement Conc. Res., 37:329-336, 2007. 
[29] Jesper Kildsgaard and Peter Engesgaard. Numerical analysis of biological clogging in two-dimensional sand box experiments. J. Contam. Hydrol., 50:261-285, 2001.

[30] J. B. Kool and J. C. Parker. Development and evaluation of closed-form expressions for hysteretic soil hydraulic properties. Water Resour. Res., 23(1):104-114, 1987.

[31] Rakesh Kumar and B. Bhattacharjee. Porosity, pore size distribution and in situ strength of concrete. Cement Conc. Res., 33:155-164, 2003.

[32] Michel Küntz and Paul Lavallée. Experimental evidence and theoretical analysis of anomalous diffusion during water infiltration in porous building materials. J. Phys. D, 34:2547-2554, 2001.

[33] Frederick M. Lea. The Chemistry of Cement and Concrete. Edwin Arnold, Glasgow, third edition, 1970.

[34] D. Lockington, J.-Y. Parlange, and P. Dux. Sorptivity and the estimation of water penetration into unsaturated concrete. Mater. Struct., 32:342-347, 1999.

[35] D. A. Lockington and J.-Y. Parlange. Anomalous water absorption in porous materials. J. Phys. D, 36:760-767, 2003.

[36] Marc Mainguy, Claire Tognazzi, Jean-Michel Torrenti, and Frédéric Adenot. Modelling of leaching in pure cement paste and mortar. Cement Conc. Res., 30:83-90, 2000.

[37] S. A. Meier, M. A. Peter, A. Muntean, and M. Böhm. Dynamics of the internal reaction layer arising during carbonation of concrete. Chem. Eng. Sci., 62:1125-1137, 2007.

[38] V. G. Papadakis, C. G. Vayenas, and M. N. Fardis. A reaction engineering approach to the problem of concrete carbonation. AIChE J., 35(10):1639$1650,1989$.

[39] Leendert Pel. Moisture transport in porous building materials. PhD thesis, Technische Universiteit Eindhoven, February 7, 1995.

[40] Bertil Persson. Self-desiccation and its importance in concrete technology. Mater. Struct., 30:293-305, 1997.

[41] S. J. Preece, J. Billingham, and A. C. King. On the initial stages of cement hydration. J. Eng. Math., 40:43-58, 2001.

[42] Anna V. Saetta, Bernhard A. Schrefler, and Renato V. Vitaliani. 2-D model for carbonation and moisture/heat flow in porous materials. Cement Conc. Res., 25(8):1703-1712, 1995. 
[43] S. C. Taylor, W. D. Hoff, M. A. Wilson, and K. M. Green. Anomalous water transport properties of Portland and blended cement-based materials. J. Mater. Sci. Lett., 18(23):1925-1927, 1999.

[44] Paul D. Tennis and Hamlin M. Jennings. A model for two types of calcium silicate hydrate in the microstructure of Portland cement pastes. Cement Conc. Res., 30:855-863, 2000.

[45] Jeffrey J. Thomas and Hamlin M. Jennings. Effects of $d_{2} o$ and mixing on the early hydration kinetics of tricalcium silicate. Chem. Mater., 11:1907$1914,1999$.

[46] F. Tzschichholz and H. Zanni. Global hydration kinetics of tricalcium silicate cement. Phys. Rev. E, 64:016115, 2001.

[47] WBCSD. The cement sustainability initiative: Our agenda for action. World Business Council for Sustainable Development, Available at http://www.wbcsdcement.org, July 2002. 


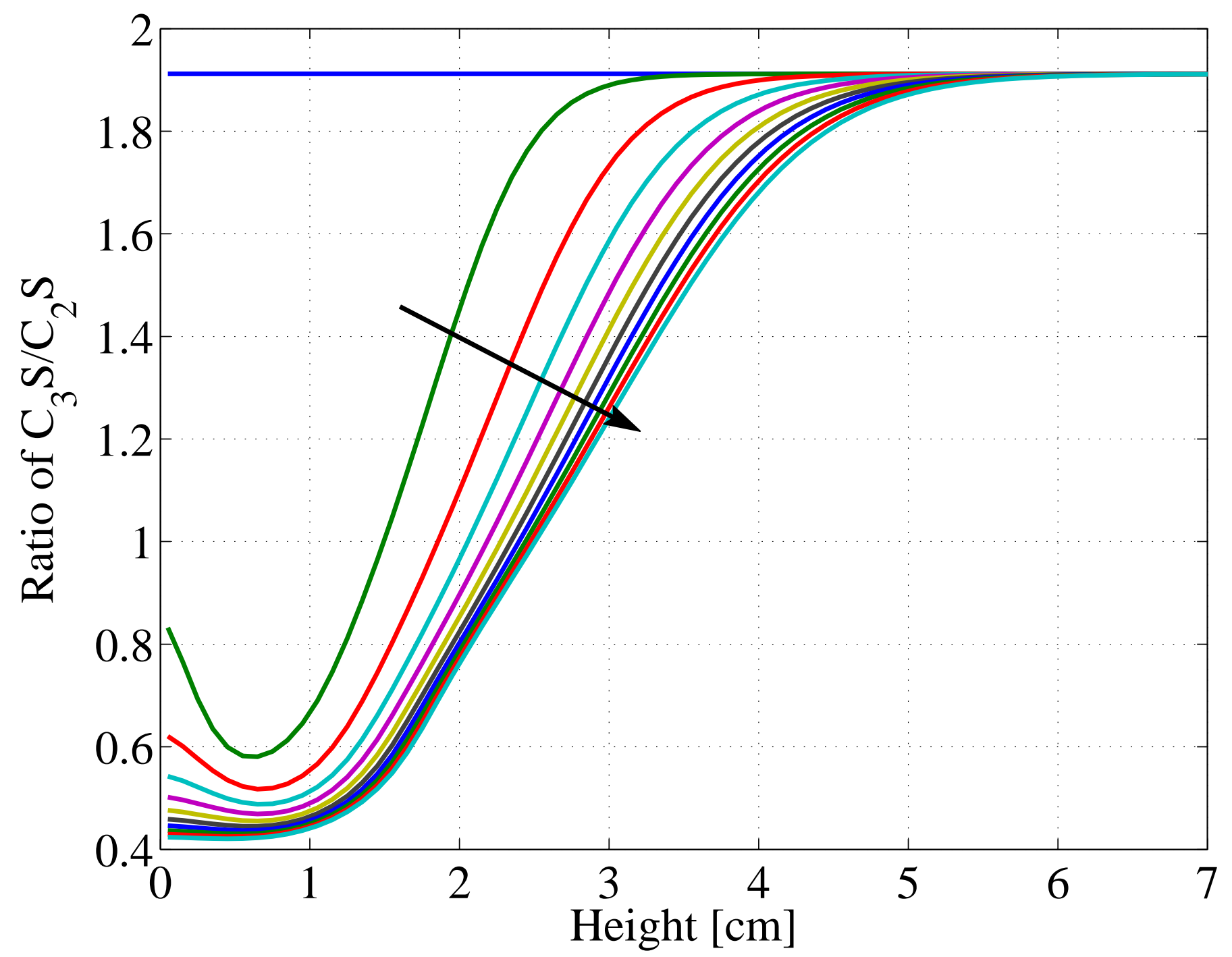




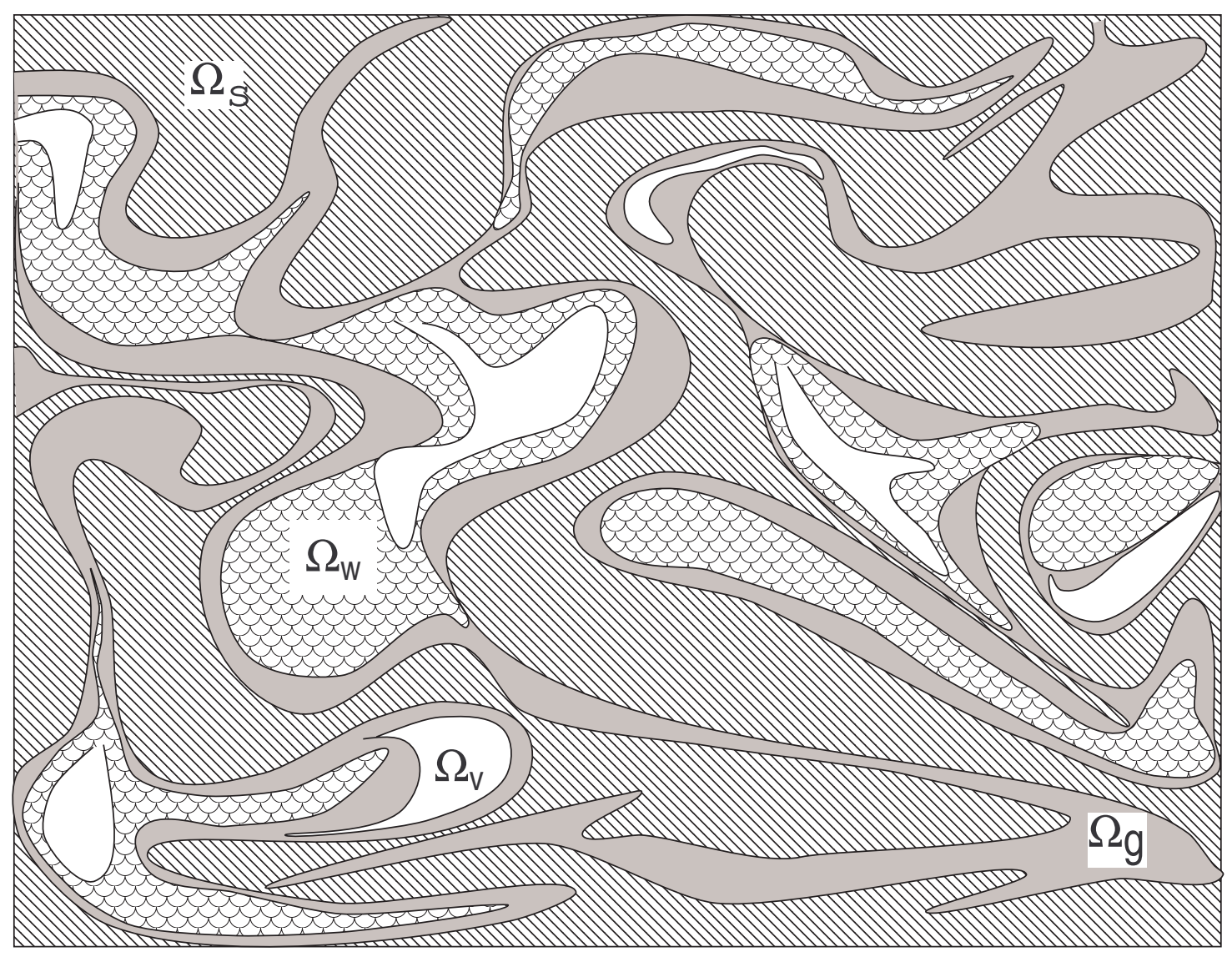

